Fecha de recepción: marzo 2020 Fecha de aceptación: abril 2020 Versión final: mayo 2020

\section{De la Bauhaus a la Materia Activa: deconstrucción y reconstrucción del Diseño en 100 años de historia}

Leandro Gejfinbein ${ }^{(1)}$ y Rejane Spitz ${ }^{(2)}$

Resumen: Si tomamos como punto de partida el surgimiento de la escuela Bauhaus, el diseño como disciplina - una conjunción de teoría, práctica y educación - completa, en 2019, un siglo de existencia. Durante ese tiempo, se ha convertido en un elemento fundamental de la sociedad contemporánea, marcado por la producción y el consumo en masa, la urbanización y la revolución en las tecnologías de la información y la comunicación. Su esencia y función originales - planificar y dar forma a todas las cosas artificiales que crea la humanidad, guiadas por una idea racionalista y determinista, en busca de soluciones definitivas - tuvieron que transformarse para satisfacer las demandas de la sociedad. Más recientemente, la demanda de contextualización, medios digitales y el desarrollo de la llamada materia activa apuntan a interrupciones aún más profundas. En este artículo hacemos una revisión histórica y crítica del diseño y, desde cuatro puntos de inflexión, proponemos una nueva mirada a los aspectos más fundamentales de la disciplina y sus desafíos para el futuro.

Palabras clave: Diseño - materia activa - digital - sociedad - transformación - ideologia.

[Resúmenes en inglés y portugués en las páginas 38-39]

(1) Director de Experiencia e Innovación de Producto en la empresa Globo, Brasil. Graduado en Comunicación de PUCRS, Master en Diseño de PUC-Rio y estudiante de Doctorado en Diseño de PUC-Rio.

(2) Investigadora y Profesora del Departamento de Artes \& Design, PUC-Rio, Brasil. Coordinadora del Laboratório de Arte Eletrônica (LAE), PUC-Rio. Doctora en Educación (PUC-Rio), Post-Doctora en Arte Electrónico (Universidad de California, Berkeley y San Jose State University). 
El diseño es la relación entre comprender y crear.

(Terry Winograd)

\section{Introducción}

Durante 100 años, la teoría y la práctica del diseño, como un área de conocimiento y un campo de actividad profesional, han evolucionado para continuar respondiendo satisfactoriamente a la necesidad de resolver viejos y nuevos problemas. Al igualar la búsqueda de eficiencia, belleza, calidad e impacto, el Diseño persigue a su ideal creador y transformador de la relación de las personas con el mundo, dando forma a todo lo que la humanidad inventa para sí misma. Desde una perspectiva histórica amplia, es posible construir un hilo común que conecte ideas fundamentales desde el establecimiento formal de la disciplina, considerando, como punto de partida, la escuela Bauhaus, hasta capítulos más recientes que amplían el espectro del Diseño. En este viaje, emancipado de su conexión genealógica e histórica con la arquitectura y su participación de apoyo como actividad instrumental a raíz de la revolución industrial que estalla en el siglo XIX, el Diseño define su papel, delimita su alcance y revela su importancia para la sociedad. Se pueden ver diferentes fases, movimientos estilísticos, enfoques de atención, reflexión y evolución en una revisión cuidadosa de la historia, impregnada de cambios contextuales de todo tipo: económicos, sociales, culturales y tecnológicos. El Diseño nace y evoluciona con los ideales, valores y cosmovisiones de su tiempo, desde el problemático panorama político y económico del período de entreguerras en Europa, hasta la posguerra y la lucha por los derechos civiles y la igualdad en los Estados Unidos, hasta las revoluciones tecnológicas desde el desarrollo de la informática, a partir de la década de 1980. Al mismo tiempo, se aprovecha el impulso de los diseñadores para adelantarse a su tiempo, inventando y dando forma a lo nuevo. Estos impulsos transformadores y evolutivos de la disciplina son vectores que impactan la forma en que diseñamos y producimos lo que se proyecta, así como las técnicas, métodos y materiales utilizados en estas producciones. Para comprender estos movimientos en una perspectiva más amplia, proponemos, en este texto, presentar el Diseño bajo el sesgo de cómo, activo o reactivo a la evolución y las demandas de la sociedad, opera las deconstrucciones y reconstrucciones de lo que es, por definición, lo que pretende ser, y de su papel en la sociedad.

Cuando contamos, bajo este sesgo, la historia del Diseño, hay cuatro rupturas que destacamos, llamadas teóricas, prácticas, digitales y materiales, que no necesariamente se suceden en cronología perfecta, ni están marcadas por los mismos catalizadores. Su importancia se debe a la forma en que confrontan lo establecido y provocan reflexiones más profundas, como puntos de inflexión, incluso si en su conjunto y diálogo también, a veces - y paradójicamente - retienen o recuperan elementos originales. Aun así, vistas de manera acumulativa, estas rupturas apuntan a una transformación más abrupta, radical y paradigmática que posiblemente caracterizará los próximos 100 años de Diseño: un diseño autónomo, responsivo, inteligente y resistente, un diseño que pulsa, respira, se transforma y se adapta, tratando de sobrevivir a las condiciones más difíciles. 


\section{Los fundamentos del diseño: lo artificialracionalista, objetivo, puro, universal}

El surgimiento del Diseño como disciplina, que combina formalmente teoría y práctica, fue considerado, en este texto, como coincidente con la creación de la escuela Bauhaus, en las primeras décadas del siglo XX. Incluso si ya había talleres diseñados para diseñar esos objetos que serían producidos en masa por la próspera industria manufacturera que revolucionó la sociedad europea en ese momento, especialmente en Inglaterra y Alemania, fue Bauhaus quien protagonizó la sistematización de lo que ahora tenemos como un campo práctico. e investigación, organizada en un programa de enseñanza que combina la teoría y la práctica del Diseño, dirigida a un fin industrial, y apoyada por fuertes ideas sociales, políticas y económicas. Lo que justifica que tomemos a Bauhaus como punto de partida es que la escuela ha cambiado profundamente su comprensión del Diseño -que antes se percibía como una actividad operativa de una línea de producción y que se convertiría en el centro de la materialización de los anhelos políticos, sociales y económicos de la sociedad. La propuesta de la escuela, fundada y dirigida en gran parte de su breve historia por Walter Gropius, era crear un centro para la formación y creación de un nuevo diseño, que rompería intencionalmente con los conceptos de la época, y dirigido a la producción industrial. Este objetivo, que puede parecer común en el contexto actual, fue revolucionario y estuvo profundamente influenciado por el contexto político y económico de Europa y Alemania a principios de ese siglo. Al ascender a un papel sin precedentes en el juego económico mundial de la época, Alemania, incluso antes de la Primera Guerra Mundial, estaba en camino de convertirse en la mayor potencia industrial y cultural por delante de Inglaterra, con el objetivo de combinar esta posición con una capacidad catalizadora. También una universalización del estilo estético y el lenguaje de un mundo que comenzaba a ser moldeado por la producción en masa.Después de la Primera Guerra Mundial, con su país devastado, este ímpetu encuentra unafuerza adicional en el sentido de que la escuela también podría desempeñar un papel importante en la recuperación económica y en el sellado de un nuevo contrato social nacional de acceso más universal a los bienes materiales, carente de privilegios y diferencias. Fue influenciado por esta idea que, en 1919, Bauhaus fue fundada en la ciudad de Weimar. La escuela tuvo una primera fase de montaje y gestión de su programa, que presuponía la unión del arte y la técnica, marcada por una creencia en el expresionismo, fruto de las ideas y el trabajo de Johannes Itten, que quería crear y desarrollar expresiones naturales y individuales. Sin embargo, es en la siguiente fase, a partir de 1923, con la renuncia de Itten e influenciado por el movimiento De Stijl, que, de manera más amplia y permanente, se consolidan las características fundamentales que aportan a la teoría del diseño y la práctica de lo que Gropius se había idealizado desde su fundación, y se vuelve aún más fuerte en los directores posteriores. Gropius afirmó que la idea de construir era una actividad social, intelectual y simbólica que tenía la intención de eliminar las diferencias entre el arte, la técnica, la industria y las personas, allanando el camino para lo que llamó una nueva estructura del futuro (Droste, 2013). El movimiento De Stijl ya argumentó que al buscar elementos primarios y básicos del lenguaje visual, sería posible romper lo que consideraban la supremacía del individuo, 
para poder crear soluciones colectivistas. Al llevar el concepto a Bauhaus, Gropius reforzó en el programa, y en sus propias clases, que buscaba denominadores comunes, formas y colores primarios como punto de partida tanto en arquitectura como en diseño. La teoría, la técnica y los productos deberían buscar un retorno al origen (ya sea desde un estado de aprendizaje o desde los elementos de construcción del diseño y el lenguaje visual) para que pudieran ser vectores de un redescubrimiento de la unidad perdida de todas las cosas (Lupton, \& Miller, 2006). El ideal de universalización impregnaba todo el programa, lo creado y la composición de lo creado, reforzado por los trabajos de Kandinsky, Klee y Albers, quienes se esforzaron por desacoplar la estética, la percepción visual y la enseñanza del diseño en formulaciones lógicas. y racional, creando modelos sistemáticos de reflexión teórica y aplicación práctica, muchos de los cuales tenemos hoy como base del diseño. En su gramática de pureza de forma y color, Kandinsky incluso propuso la relación, que se ha hecho famosa, que los triángulos son amarillos, los cuadrados son rojos y los círculos son azules (Lupton y Miller, 2006).En su obra Punto y Línea sobre el Plano, Kandinsky propone organización y método para crear una nueva ciencia del arte ${ }^{1}$ (Kandinsky, 2012). Estos son ejemplos que, independientemente de si tienen sentido o no, dada la relativización que es más característica de nuestro contexto actual, muestran claramente la importancia de una búsqueda teórica y ensayos prácticos impulsados por creencias y principios racionalistas y universalistas. Otro ejemplo que arroja luz sobre estos conceptos, y cómo se han traducido en la práctica, es la creación atribuida a Herbert Bayer de la tipografía adecuadamente llamada Universal. Es el resultado, como dijeron los voceros de la escuela en ese momento, de un nuevo código de valores visuales basado en la creencia en un futuro de leyes universales de la razón que liberan a las personas de los confines de la cultura tradicional (Lupton y Miller, 2006). Para Bayer, la cultura es artificial y solo la razón y la ciencia son puras. La simplicidad de las formas geométricas sería socialmente liberadora, ya que no tenía ilusión ni un estilo aristocrático oculto. Las formas serían "desnudas" en significado, vacías de ideología cultural, como lo fueron las máquinas. Creía en un diseño que sería el resultado de elementos asumidos como naturales, sin la influencia de factores sociales, económicos y políticos, como si de hecho hubiera una naturaleza dada y no una idea de universalización tomada por natural y propria del contexto ideológico que se estaba extendiendo en Alemania en ese momento, especialmente en Weimar, lugar de nacimiento de la Bauhaus.

Con la disolución de la escuela en 1933, impuesta por el ascenso del régimen nazi, varios de sus maestros emigraron a los Estados Unidos y se establecieron en Chicago. La ciudad ya albergaba a un grupo de arquitectos influenciados por la idea de la liberación del adorno, a favor de proyectos que luchaban por la racionalidad, la eficiencia y la visión aséptica de que la complejidad debería simplificarse y lo inútil debería eliminarse. Se dice que Louis Sullivan, uno de los arquitectos estadounidenses más influyentes de la época, con sede en Chicago y mentor de Frank Lloyd Wright, creó la famosa expresión "la forma sigue a la función". El grupo de salida de Alemania funda New Bauhaus en Chicago, que, en comunión con el movimiento racionalista ya presente en la arquitectura, está impulsando y diseñando para el mundo, de manera decisiva, el modernismo, que tuvo su apogeo en las décadas de 1950 y 1960, pero está muy presente como una influencia en el Diseño y la Arquitectura hasta nuestros días. 
La universalización, la racionalización, el tecnicismo, la eliminación del espacio de diferencias y particularidades, la eliminación de la individualidad y la búsqueda de un marco teórico que funde la naturalidad y la pureza de esta idea marcan el surgimiento del Diseño. Los decoupagestécnico-científicos de cuestiones estéticas y de percepción dan lugar a técnicas, métodos, fundamentos y criterios de calidad, basados en una fuerte visión específica del mundo y la ambición de definir cuál debería ser el papel del Diseño en este contexto. Así nace el Diseño.

Es interesante señalar que no hay necesariamente en Bauhaus, explícitamente, una declaración sobre la eternidad y la inmutabilidad del producto final del Diseño. Sin embargo, consideramos que esta idea estaba presente, ya que era parte de este nuevo propósito "sagrado" del Diseño, esta nueva idea platónica de una búsqueda para el cumplimiento de la perfección pura, el estado fundamental y la composición. En esta creencia yace la búsqueda de un mundo nuevo, pero desea ser definitivo. Esta fe en lo definitivo será confrontada más tarde y, a veces, deconstruida, al menos en sus extremos. Sin embargo, debe enfatizarse que tal vez, si no hubiera habido tal ambición y arrogancia en ese momento, el Diseño no habría logrado la influencia y la repercusión que había logrado, y no habría alcanzado la disciplina en la que se ha convertido, y su importancia ha persistido durante tanto tiempo. Incluso hoy, incluso después de una sucesión de ideas, personas, tecnologías y concepciones divergentes que han curvado este camino una vez idealizado de precisión rectilínea, parece bastante razonable decir que vivimos y viviremos durante mucho tiempo influenciados por gran parte de lo que Bauhaus ha forjado.

\section{La ruptura teórica: el diseño es entre el hombre y el mundo, es contextual, dinámico, impredecible y singular.}

En un editorial de la revista científica Design Studies de 2007, Nigel Cross revisa críticamente el hito de 40 años en la investigación de Diseño desde la creación en la década de 1960 de la Design Research Society, que tenía como objetivo promover el estudio y la investigación del proceso de Diseño en todos sus campos. A partir de esto, hay un esfuerzo por consolidar el Diseño como un campo académico de estudio, elevado al estado de la ciencia. En su texto, Cross resume esta trayectoria, situando este punto de partida contextualizado por el escenario de la época, donde el trabajo para lograr este objetivo estaba contaminado por una visión excesivamente racionalista del diseño, de la cual Cross es muy crítico. En una versión más metódica e intensa, en comparación con el racionalismo inaugurado en la idea y la enseñanza de Bauhaus (donde la conjugación con el arte jugó un papel importante), un conjunto de textos de los años 50 y 60 tomaron el proceso de diseño como algo que debería buscar la máxima adaptación al rigor metodológico típico tanto del contexto industrial como de los métodos científicos. Vale la pena mencionar la creciente influencia en el momento del desarrollo de otro fenómeno de profundo impacto transformador de la sociedad en el siglo XX: la moderna tecnología computacional. En una formulación simple, el objetivo del Diseño (y el rol de los diseñadores) se veía como o de resolución de problemas y, en la referencia crítica de Cross, los problemas se resolverían a través de métodos que fueran eficientes, ya que eran el resultado de enfoques técnicos y racionalistas. Y matemáticas No es casualidad que las 
formulaciones matemáticas tengan un alto grado de abstracción que influyó en la idea de lo que fue el Diseño, y de cómo llegar a un buen Diseño. Además, el Diseño era un medio garantizado para alcanzar algo, y en eso algo residía el valor, la forma y la creación material que resultó de la actividad. El Diseño se consideraba una extensión de la Ingeniería y se requería que abarcara rigidez y descripción en su proceso, pasos de validación y reproducción de sus resultados.Para hacer un diseño correcto, fue necesario hacer del Diseño un proceso científico, rígido y replicable, siendo el resultado final simplemente el resultado de la buena ejecución de la secuencia de los métodos de Diseño.

Desde finales de la década de 1960 en adelante, preguntas sobre este punto de vista han comenzado a tomar forma y ganar fuerza, deseando reemplazar estas ideas con una teoría que mantenga el objetivo - elevar el Diseño a un campo de estudio y ciencia - pero a partir de una esencia propia del Diseño, de una tipificación específica del problema de Diseño, diferente de otros campos de la ciencia. Para esta nueva propuesta, sería urgente crear una forma de diseño como ciencia y actividad singular, y dicha singularidad caracterizaría el Diseño mismo, el pensamiento de Diseño (design thinking), en su función y lugar único en el mundo. La reflexión tenía como objeto de análisis el proceso, el diseño, pero, a pesar de esto, la ruptura (y la agitación en el entorno académico) se limitaba al alcance teórico. La influencia de estas nuevas proposiciones y este debate, dentro del alcance de la práctica, se restringió a movimientos de cambios estilísticos, como en el surgimiento del deconstructivismo y el posmodernismo, que rechazaron, por expresión estética, la perspectiva racionalista. Lo que realmente cambió fue un marco conceptual completo que estaría marcado por contornos más humanistas, la búsqueda de la unicidad y la conciencia de que habría una naturaleza dinámica y menos técnica del diseño. El Diseño se defendió como una actividad de proceso reflexivo-práctico implícito, que exigía un rescate de los contornos artísticos de expresión de un repertorio individual y contextual, de intuición, de aspectos propios para tratar problemas caracterizados por la incertidumbre, inestabilidad, individualidad y conflictos.

Herbert Simon fue un teórico emblemático en este momento de la historia. Su obra más famosa, "Las Ciencias de lo Artificial", escrita en 1969, dio lugar a interpretaciones ambiguas y choques acalorados, pero fue una contribución fundamental a las deconstrucciones y reconstrucciones del Diseño en esta ruptura teórica que hemos descrito. El autor se centra en la tarea de definir el Diseño como ciencia: la ciencia por excelencia de lo artificial en oposición a la ciencia de lo natural. Aunque el trabajo ha hecho mucho para difundir el enfoque racionalista y mecanicista que Cross y otros teóricos han criticado, el autor también debe ser acreditado con gran parte de la forma única y sin precedentes en la que se describe, define y caracteriza el problema del Diseño mediante un replanteamiento de aspectos del objetivo final del Diseño. Por lo tanto, si, por un lado, el texto se dedica en gran medida a proponer y describir un amplio conjunto de métodos, tratados en una extensión, preciosidad, granularidad y derivación de conceptos de las ciencias exactas, la psicología cognitiva (una visión técnica). Por otro lado, fue precisamente este extenso y detallado conjunto de métodos cuantitativos lo que desencadenó en Simon una conciencia y claridad con respecto a la complejidad, variabilidad, dinamismo y particularidad de los pro- 
blemas que el Diseño propone resolver. Su definición de diseño, un conjunto de acciones que tienen como objetivo cambiar las situaciones existentes a las preferidas (Simon, 1996), trae implícitamente una libertad y una amplitud de comprensión de lo que el diseño debe ser disfrutado incluso por sus críticos en las décadas siguientes. La definición agnóstica de materialización, del fin del diseño como objetos, resalta el carácter transformador y creativo del diseño, desde la comprensión de la realidad (o problema) que se propone resolver. Lo interesante de la artificialidad, dice, es que está relacionada con una doble complejidad, porque estos son sistemas complejos que existen en entornos complejos. El diseño, como algo artificial, existe en la relación entre el hombre y el mundo, y la tarea de diseñar y construir un artefacto necesita considerar un sistema complejo donde varias fuerzas actúan y reaccionan entre sí. Este comportamiento a menudo se asocia, en sus textos, con aspectos adaptativos y evolutivos, que también son características intrínsecas de lo que es artificial. Simon también argumenta que un artefacto debe verse como una interfaz, el punto de encuentro entre lo que él llama el ambiente interno y el ambiente externo. La lógica del Diseño es un proceso dinámico de búsqueda de alternativas, y cuyo resultado a alcanzar siempre es suficiente, no necesario, presupone la exploración de diferentes caminos en paralelo, así como una racionalización en el uso de los recursos. En esencia, es un enfoque que busca el mejor resultado posible para un problema que, en teoría, tiene, y nunca tendrá, una solución única, ni una que sea perfecta. Esta proposición confronta directamente la creencia de que existe en las formas y sus composiciones una naturaleza pura, sagrada y fundamental. El autor también declarará que el estilo de lo que se crea está profundamente influenciado por la forma en que se lleva a cabo el proceso de Diseño y, por lo tanto, no hay un final absoluto, sino diferentes resultados posibles, consecuencias directas de las elecciones del proceso. Simon también comenta sobre la importancia de la representación en el proceso de Diseño, inspirado en dotaciones matemáticas, y afirma que desempeñarán un papel central en el futuro de la teoría del Diseño, ya que podrán revelar, hacer visibles, las decisiones y la lógica detrás de decisiones, que tienen un impacto directo en cómo se continuará desarrollando dicha solución. Después de detallar su propuesta de lo que debería ser la Ciencia del Diseño, Simon dedica un capítulo a la discusión de los cambios en esta propuesta cuando el Diseño debe considerarse como una herramienta para resolver problemas a escala social, reforzando la naturaleza compleja y dinámica de los problemas de Diseño. El autor cita aspectos tales como: proyectar sin que el objetivo final sea claro, oproyectar asumiendo cambios continuos durante todo el proceso de diseño e implementación. En el límite del concepto, lo que se debe proyectar es solo el comienzo de algo que no se puede predecir con certeza a dónde llegará. Al concluir el capítulo, el autor pide una conciencia necesaria del hecho de que no hay límite en el mundo para la diversidad, no hay certeza sobre la evolución, y el diseño inevitablemente tiene que lidiar con eso, en oposición diametral a la ideología del Diseño que se inauguró en la Bauhaus. Como ya comentamos, sin embargo, la propuesta de Simon es ampliamente criticada, particularmente en la forma en que propone reorganizar los procesos y métodos para tratar estos problemas que él define de manera tan singular, y sobre todo por un conjunto de autores que se niegan a esta visión tomada de técnicas computacionales, metodologías 
científicas y teorías de gestión, que persiguen la ciencia del Diseño a través de este prisma técnico. Bruce Archer es otro de esos críticos y, en 1979, propone una divergencia. En la teoría del autor, el diseño se presenta como un tercer elemento, no necesariamente una ciencia. El diseño existe en comunión junto con Humanidades y Ciencia, como en un sistema de fuerzas que se interrelacionan, pero son diferentes en sus propósitos, justificando que cada uno busque sus propios métodos (Archer, 1979). Otros autores, como el dúo Horst Rittel y Melvin Webber (Rittel, Webber, 1973), a los que también hace referencia Nigel Cross, se oponen a la idea racionalista de enfrentar el problema y a la solución de Diseño definiendo lo que llamaron wicked problems (problemas perversos, en traducción libre). En un texto de 1973, preocupado por advertir sobre una teoría de planificación que se ocupa de problemas en una sociedad de creciente complejidad, conflicto y diversidad, los autores se adhieren a la definición del problema de Diseño en un rechazo de la primacía de la eficiencia como el objetivo de resolver cualquier problema. Los autores también critican lo que llaman la arrogante confianza profesional de los diseñadores como solucionadores de problemas (cualquier problema), dada la deficiencia en cómo se definen y localizan los problemas a escala, y la incapacidad del enfoque racionalista para ofrecer formas adecuadas de tratar tal complejidad. Los investigadores afirman que los problemas de planificación (entendidos aquí como problemas de Diseño complejos a escala) son intrínsecamente perversos, en oposición a los problemas a los que los científicos e ingenieros estarían acostumbrados a tratar, que los autores se definen como problemas dóciles. A partir de ahí, los autores definen 10 características de wicked problems, problemas perversos con los que los diseñadores tienen que lidiar, y donde uno nota la ambigüedad con la que se dirige la crítica a Simon: 1) En el Diseño no existe una formulación definitiva del problema. y las actividades de comprensión y resolución son concomitantes e influyen cíclicamente entre sí; 2) no hay un final claro, un criterio claro que garantice que se ha encontrado la solución final y definitiva; 3) las soluciones no pueden clasificarse como verdaderas o falsas, sino, en el mejor de los casos, como buenas o malas; 4) No hay pruebas definitivas que validen problemas perversos; 5) cada solución implementada genera consecuencias que alteran el problema inicial, haciendo que los modelos de prueba y error no estén exentos; 6) no hay un número establecido de posibles soluciones que puedan contarse o describirse definitivamente; 7) cada problema es esencialmente único en la medida en que no hay generalizaciones posibles como clases que determinan el comportamiento de todos sus miembros; 8) cada problema es potencialmente un síntoma de algún otro problema, como en un sistema donde, en cierta medida, todo se relaciona; 9) al definir un problema, su representación puede tener lugar de innumerables maneras, influyendo en la definición del problema en sí, lo que le da a la actividad una subjetividad inherente; 10) el diseñador no tiene derecho a cometer errores, no en el sentido de suponer que su objetivo es llegar a una verdad absoluta (que no existe), sino en su necesidad, en su papel, de ser consciente del impacto y consecuencias de lo que se generará (Rittel y Webber, 1973).

Como una contribución más reciente a este avance teórico están las ideas del autor Donald Schön. En un artículo de 1993 titulado Learning to Design and Designing to Learn, Schön reflexiona sobre relacionar directamente su teoría de la práctica reflexiva (publicada diez años antes en un texto de 1983) con el Diseño. El texto comienza con referencia a Herbert Simon, donde Schön declara estar de acuerdo con su amplia visión del Diseño y las 
ciencias artificiales, pero no está de acuerdo con la llamada visión instrumental del proceso de Diseño, que define como una búsqueda heurística de un conjunto de limitaciones Para Schön, el Diseño es una transacción, un acto de intercambio entre el diseñador y los materiales en una situación de diseño. El tema de los materiales es fuerte en su discurso: el autor recuerda la importancia de la materialidad en el Diseño, que, como hemos visto, pierde su centralidad en las proposiciones y agitaciones teóricas descritas aquí, y algo que era demasiado importante en los fundamentos de Bauhaus. Incrustado en esto hay una crítica no solo de la visión instrumental, científica incorporada en Simon, sino también una crítica del valor de la abstracción per se en una actividad que está esencialmente relacionada con la creación y / o transformación de materiales y - lo que es central para su idea - donde la práctica lleva a la reflexión, y la reflexión a la práctica, que son inseparables entre sí. El autor enfatiza la apreciación en el proceso de apreciación sensorial y activa de los profesionales del Diseño en el hacer Diseño, independientemente de la naturaleza del que se esté generando. Es una conversación donde el diseñador ve, actúa, ve de nuevo, actúa de nuevo. Desde el concepto de "mundo del diseño" (que puede entenderse como el problema del diseño), Schön también hace una defensa aún más contundente que este "mundo" donde un diseñador actúa es único, particular para un diseñador o comunidad en particular de diseñadores, y de donde toma lugar ese Diseño. Y cuanto más innovador queremos que sea el resultado, más único es este "mundo" en el que debe trabajar el diseñador, influyendo fundamentalmente en cómo se realizará este trabajo. Curiosamente, vemos el vínculo en las ideas de práctica reflexiva de Schön y la propuesta de enseñanza central de Bauhaus de ciclos interconectados de teoría y práctica, como el alma de la actividad de Diseño. El profesor de la Bauhaus, Josef Albers, definió el curso como aprender haciendo, declarando en una conferencia dada en 1929 (Wingler, 1976) que la mejor educación es la propia experiencia de jugar, experimentar, inventar y reinventar desde el contacto con los materiales. Al final de cada clase, se alentó a los estudiantes a reflexionar en grupo sobre lo que hicieron y a justificar las decisiones tomadas.

Todo este movimiento teórico, aunque desde diferentes perspectivas, se centró en el proceso y dio como resultado una mirada más cercana a las técnicas y métodos, que fueron detallados, organizados o reorganizados, sistematizados al extremo, mientras sostenían que esta disciplina necesitaría adoptar un dinamismo, complejidad e individualidad inherentes a los problemas que el Diseño tiene que enfrentar y resolver a través del Diseño. En relación con las creencias de la tradición modernista que se originó en la Bauhaus, estos son los avances más significativos traídos por estos autores, rompiendo con una visión totalista y racionalista de encontrar denominadores comunes por los cuales se darán soluciones definitivas, así como métodos definitivos de llegar a ellas. Las ideas anteriores, de planificación, pureza y eternidad de formas, de simplificación como una forma de abordar las complejidades, se enfrentan con la noción de que el problema del Diseño es abierto, dinámico, impredecible y esencialmente evolutivo, y que exige un fuerte entusiasmo creativo continuo. Es decir, el Diseño revela, como ideal teórico, la solución y la realización de algo que está, y continuará, en movimiento, incluso si su resultado final práctico sigue siendo un elemento permanente. También se intentó descifrar y describir cómo eran las formas de pensar, actuar y trabajar de un diseñador, como un reflejo de una nueva perspectiva de pensar el problema y colocar el Diseño elevado al nivel de la ciencia. Por lo tanto, independientemente de si se ha logrado 
el objetivo de llevar el Diseño a una ciencia, o si estas teorías han modificado o presentado directamente nuevos modelos prácticos aplicables para romper con los diseñadores, se ha producido la ciencia delDiseño, tomando Diseño como un objeto en el que se ha puesto demasiado esfuerzo en descifrarlo, describirlo, problematizarlo y entenderlo mejor.

\section{La ruptura de la práctica: las herramientas que transcodifican y revelan una nueva apariencia y un nuevo hacer}

Aunque la ruptura teórica enfrentó los fundamentos del diseño en su idea racionalista original, reemplazándolos con una nueva forma de pensar sobre la naturaleza del problema y el propósito del diseño, en la práctica del Diseño no había, necessariamente, una innovación profunda directamente relacionada con estas ideas. Dicha innovación se revela a partir de la llegada de la tecnología computacional - y su lógica intrínseca - en el campo del Diseño. Asumiendo una máxima generalizada de que solo la tecnología puede hacer frente a la complejidad de un mundo que la tecnología misma ha producido, ese también sería el origen y la causa de la ruptura de la práctica.

No obstante, es una ironía, debido a la influencia e impactos generados por la adopción de la tecnología computacional en los procesos de diseño, que ella ha sido ignorada - o incluso rechazada, como hemos visto - desde el punto de vista teórico, con excepciones ocasionales. En su tratado de 1969 sobre La Ciencia del Artificial, Simon (1993), además de tener su propuesta de método fuertemente influenciada por la lógica computacional, realizó análisis directamente sobre el uso de las computadoras en los procesos de Diseño y, por lo tanto, puede considerarse un precursor del impacto que esto podría (y en su opinión, debería) representar. Entre 1969 y principios de la década de 1970, Nicholas Negroponte, un arquitecto que se convirtió en un notorio tecnólogo, fundador del MIT Media Lab, también escribe dos textos de importancia histórica para reflexionar sobre el uso de estas nuevas tecnologías en los procesos de Diseño, desde una perspectiva visionaria, aunque solo en concepto, dado que en ese momento no había tal capacidad computacional, sensores, procesamiento de red y aprendizaje automático para lograr lo que él imaginó (Negroponte, 1969).

Pero desde finales de la década de 1970, con la evolución y la masificación de la tecnología de la información y la entrada de computadoras en diversos campos de actividades de conocimiento y producción, existe un uso creciente de la informática en los procesos de Diseño. Luego, los objetos y entornos físicos tienen su materialidad transcodificada en representaciones digitales y procesos computacionales, lo que comúnmente se conoce como Computer-Aided Design (CAD), o diseño asistido por computadora. La adopción de estos procesos trae ganancias de eficiencia y escala, especialmente para resolver problemas complejos, tan explorados en teoría por Simon y otros autores de la ruptura teórica. La adopción de estas herramientas, sin embargo, no se limita a contribuir a hacer mejor y más rápido lo que ya se hizo, sino que tiene un impacto en la forma en que actúa el diseñador, ya que su actividad está mediada por una herramienta que es capaz para cambiar el aspecto y la forma de actuar sobre el Diseño. Las capacidades de simulación y manipulación digital, antes impensables, de la máquina tienen el poder de revelar nuevas perspectivas 
sobre el ojo atento y creativo del diseñador, o, como diría Schön, nuevas formas de realizar transacciones con materiales. Tales simulaciones digitales inevitablemente arrojarán luz sobre decisiones que tal vez nunca se tomen antes de estas posibilidades. Los cálculos precisos también respaldan la toma de decisiones sobre el umbral, que tal vez no se podría considerar porque se supondría que no era viable antes de usar estas herramientas.

Desde entonces, el uso creciente de la tecnología informática en los procesos de Diseño ha cambiado significativamente la forma en que se practica esta actividad. En su etapa más reciente de evolución, hemos llegado al llamado Diseño Paramétrico, un punto de madurez tan significativo y transformador en la forma de proyectar y la forma en que los diseñadores piensan sobre el diseño que, mucho más que solo herramientas, tiene profundas consecuencias en una nueva lógica que emerge de la nueva práctica, el estilo de lo que se crea y los procesos de producción (destacando el advenimiento de la impresión 3D). En un artículo que aborda estos impactos, Rivka Oxman (2017) argumenta que el Diseño Paramétrico, más que la aplicación de un conjunto de tecnologías y técnicas computacionales, cambia profundamente el pensamiento en el proceso de hacer Diseño. La autora realiza un análisis comparativo, comenzando por la consolidación de los diversos procesos mentales y etapas de decisión y creación involucradas en el diseño, a partir de los años 60, en trabajos que reúne y clasifica bajo el apodo design thinking. Basado en una trayectoria evolutiva, parte del pensamiento de design thinking pasa por el pensamiento de diseño computacional, y finalmente llega al pensamiento de diseño paramétrico, donde muestra cómo Diseño Paramétrico - y todas las tecnologías que se pueden agrupar bajo ese concepto, tales como aprendizaje automático, inteligencia artificial, diseño de algoritmos, autogeneración y automatización - modifique, reemplace y / o refine estos viejos procesos mentales. Oxman luego propone lo que él llama PDT - Parametric Design Thinking (pensamiento de diseño paramétrico). El autor afirma que desde el primer período de los modelos de diseño asistidos por computadora se puede observar una transición sustancial en la naturaleza de los métodos y procesos de Diseño. Además, también hay un cambio en los requisitos profesionales, el nivel de conocimiento, las habilidades, los principios conceptuales y los modelos de diseño de un diseñador cuando necesita operar en una nueva relación hombre-máquina en la actividad de Diseño. La capacidad de desarrollar algoritmos de pensamiento y escritura algorítmicos, en opinión de Oxman, se convierten en componentes fundamentales para el ejercicio del diseño.

Citando a Reas y Fry, Oxman reitera la afirmación de los autores de que un diseñador deja atrás un proceso de elección de objetos individuales, trabajando para crear una serie de todas las posibilidades de diseño existentes para un problema dado, que ellos llaman "reflexión exploratoria". Podríamos pensar que esta es una realización práctica de lo que Simon había abogado en teoría. Al definir el Pensamiento de Diseño Paramétrico, Oxman dice que el "diseñador diseña el código de un esquema paramétrico en lugar de diseñar el diseño de un objeto. “(Oxman, 2017, p. 11). La formulación del esquema paramétrico para el autor, la base del diseño - es una capacidad cognitiva inherente a la creatividad de este nuevo profesional del Diseño, un diseñador computacional.

El autor también comenta sobre cómo el Diseño Paramétrico modifica los procesos de diseño tradicionales que se ocupan de los ciclos iterativos, como el análisis binomial-síntesis y el trinomio generación-representación-evolución, así como la reflexión en acción (lo 
cual, refiriéndose a Schön, sería la práctica-reflexiva). Dichos ciclos presuponen operaciones visuales de dibujar y rediseñar un objeto, que en el Diseño Paramétrico ocurre a partir de cambios de reglas y asociaciones de algoritmos, que no presuponen necesariamente una relación sensorial directa con lo que se está modificando.

Todos estos aspectos cambian significativamente el hacer, al tiempo que ofrecen algunas soluciones que, de hecho, en la práctica, se vuelven capaces de lidiar con los problemas de diseño dinámico y complejo que los procesos tradicionales no pudieron lograr. Y a medida que avanza la aplicación de los recursos computacionales en el Diseño, responden más a la demanda de dinamismo y singularidad, que en teoría confrontaba el racionalismo en el Diseño, pero que, paradójicamente, se realiza en la práctica a partir del impacto de una representación racionalista en esencia: la evolución de la informática. En comparación con los fundamentos lanzados en Bauhaus, también hay una redención importante y notable: en esta nueva lógica computacional, se supone que la práctica del Diseño depende de un profundo decoupage de las reglas, materialidades, composiciones y efectos del Diseño, de las preguntas formales. y la estética, a la que los famosos miembros de la Bauhaus, como Kandinsky, Klee y otros, ya mencionados, estaban tan dedicados.

El Diseño Paramétrico y el uso de las máquinas contribuyen a alcanzar un nuevo nivel de diferenciación, celebrado sobre todo en arquitectura. Patrik Schumacher (2009) argumenta que el movimiento impacta de tal manera en la forma de hacer y pensar el Diseño, especialmente para la arquitectura y el urbanismo, permitiendo resultados inéditos y tan únicos en comparación con lo que ha sido posible hasta hoy, que puede considerarse incluso definiendo un nuevo paradigma de estilo - parametricismo - siendo este el sucesor del modernismo.

\section{La ruptura digital: interacción como premisa y código como materia}

En el capítulo anterior discutimos cómo la tecnología informática vincula el Diseño como una herramienta poderosa en su proceso. Pero esta es solo una de las dimensiones de impacto. En esta tercera ruptura, lo digital se convierte en un importante fin del Diseño, que convencionalmente se llama Diseño de Interacción.

Depende del Diseño de Interacción diseñar artefactos digitales, como servicios, productos, interfaces y entornos de software. La disciplina se deriva de la reflexión provocativa que los autores, los profesionales del Diseño y los investigadores estaban haciendo en la década de 1980 en el contexto del desarrollo de la industria informática en Silicon Valley, cuando se dan cuenta de la escala de la revolución que la computación en masa estaba a punto de provocar. Advierten sobre la necesidad de comenzar a diseñar estos nuevos equipos, especialmente software, orientados a una comprensión más amplia del papel de estas máquinas en la sociedad, su significado para las personas, sus formas y propósitos de uso, más allá de la perspectiva de las Ciencias Exactas.

Uno de los defensores más activos del Diseño en ese momento - Terry Winograd - no era diseñador, sino matemático y lingüista, y ganó notoriedad por desarrollar la primera interfaz de lenguaje natural para computadoras en la década de 1970. Publicó un trabajo temprano en defensa de diseño de computadora en 1986 (Understanding Computers and 
Cognition: A New Foundation for Design), y se dedicó a influir y hacer que la industria y la academia sean conscientes de este nuevo aspecto, criticando la forma en que se consideraba a los usuarios en el proceso en ese momento, en función de un conjunto de métodos de prueba y análisis restringidos a un campo que se ha denominado convencionalmente $\mathrm{Hu}$ man Computer Interaction (HCI). En la visión crítica de Winograd, ver al humano desde la perspectiva de la máquina, no la máquina desde la perspectiva humana, era abordar el tema desde una perspectiva demasiado racionalista. Winograd creía que las computadoras solo serían más relevantes para la sociedad si los ingenieros entendieran la importancia del Diseño y pudieran atraer a profesionales del diseño preocupados por los aspectos humanos de la experiencia.

Después de haber obtenido el respaldo de compañías, profesionales e investigadores influyentes, y anclado en historias de éxito como la de Apple - una empresa cuya creencia en el diseño y el cuidado de un amplio proyecto de experiencia centrada en las personas ha demostrado tener un valor de mercado decisivo - esta perspectiva ganó fuerza a partir de la década de 1990 en adelante, con la publicación de About Face (1995), un trabajo de varios autores dirigido por Alan Cooper, sobre los elementos esenciales del Diseño de Interacción. (Cooper, Reimann, Cronin y Noessel, 2014).

En la década de 2000, ya había una consolidación y profesionalización de la disciplina. Publicado en 2006, Designing Interactions es el trabajo más referenciado en el campo, teniendo como organizador a Bill Moggridge, diseñador, educador, investigador, fundador de IDEO y quien acuñó el término Diseño de Interacción en la década de 1980, trabajando en Silicon Valley, California. Como telón de fondo de este movimiento, a partir de la década de 2000, tenemos el fenómeno de la masificación de la informática personal, redefiniendo los límites de la permeabilidad de los productos y servicios digitales y de red para el consumo masivo en la vida cotidiana de nuestra sociedad contemporanea, apalancada, sobre todo, por la emergencia de los teléfonos inteligentes. En su definición, Moggridge (2006) afirma que la disciplina que él llama es el equivalente del Diseño Industrial, pero se aplica al mundo virtual, donde asume sus propios matices y especificaciones. Por lo tanto, esta propuesta combina técnicas tradicionales y principios de Diseño, ya bien establecidos, con una nueva materialidad - el medio digital - así como la lógica y los lenguajes de la informática y las propiedades de la relación del hombre con estas máquinas. A medida que la tecnología evoluciona, el Diseño de Interacción se vuelve más complejo. En este período, presenciamos, en la dimensión del hardware, una proliferación de tipos de dispositivos computacionales con los que interactúa, el aumento exponencial de la potencia de procesamiento, las posibilidades de los componentes y materiales de estos dispositivos, de nuevos sensores, complementados por pantallas más y más precisas y sofisticadas. $\mathrm{Pa}$ ralelamente, las nuevas fronteras de la experiencia de interacción son introducidas por las evoluciones de la capa de software, como la computación en la nube, la inteligencia artificial y el aprendizaje automático. También estamos avanzando hacia un nuevo ciclo de masificación de entornos y objetos totalmente digitales, que simulan y aumentan la realidad con fidelidad y, por así decirlo, un realismo cada vez más sorprendente. El contexto de las interacciones evoluciona a sistemas con comportamientos más dinámicos, programados para adaptarse completamente al contexto de uso y usuario. Sin embargo, dos características permanecen y definen la esencia del diseño de interacción, y se basa en 
estas propiedades que está justificado hacer de este movimiento uno de los cuatro puntos de ruptura descritos en este documento: la primera característica - que está en el nombre de la disciplina - es la interacción como foco del Diseño; la segunda tiene que ver con la materialidad - o la no-materialidad - de los objetos que se crean, hechos de códigos.

No es casualidad que el Diseño aplicado a lo digital, cuando se bautiza, se convirtiera en Diseño de Interacción. La interacción es inherente al artefacto que se diseña y produce. La característica interactiva de los sistemas e interfaces digitales es lo que distingue fundamentalmente este tipo de diseño de todos los demás. Aunque conceptualmente podemos admitir que siempre hay un nivel de interacción más o menos explícito con cualquier objeto y entorno, la interacción digital no es solo uno de los aspectos a ser contemplados, sino su esencia, desde la cual las otras dimensiones se considerarán en función de los objetivos, posibilidades y límites de los primeros. Dan Saffer (2010) describe la disciplina como relacionada con el pensamiento y la definición del comportamiento de los productos digitales; Interaction Design se ocupa de comportamientos, transacciones entre entidades, intercambio de información, bienes, servicios, sentimientos, emociones y sensaciones, y abarca formas de interacción mucho más indirectas que las manipulaciones a las que estamos acostumbrados, como las que suceden gestos, voz y presencia. Para buscar precisión en su definición, Saffer da una breve historia de lo que considera la necesidad de diseñar interacciones, mucho antes del advenimiento del Diseño de Interacción tal como se presenta aquí, reforzando, al final, que la diferencia está en el momento en que el foco se convierte en software, no en objetos físicos (hardware). Sugiere que Interaction Design requiere una comprensión de la filmografía, el teatro, la biología, la lingüística e incluso la terapia (subrayando el tema de la empatía, ya que la psicología más amplia como ciencia se ha vinculado mucho a la perspectiva de factores humanos, muy racionalista).

David Rokeby, un conocido artista canadiense en las artes electrónicas, reflexionando sobre su propio trabajo y los aspectos de tratar con los medios interactivos, en un ensayo publicado en 1995, llama la atención sobre la fuerza transformadora que trae la posibilidad de trabajar con medios interactivos - para quienes los crean, así como para quienes los usan y prueban. Define los medios interactivos como un medio que refleja las consecuencias de nuestras acciones y decisiones de vuelta al interactor, y hace una analogía con los espejos, a través de los cuales nos comunicamos con nosotros mismos, en el sentido de que un espejo no solo refleja sino refracta lo que viene a él, devolviendo algo transformado y procesado. Un sistema interactivo responde al interactor, que responde de nuevo, y esto se repite continuamente entre el sistema y el interactor, y donde las acciones (y todas las dimensiones involucradas en el diseño de este artefacto digital) se multiplican hasta el infinito como dos espejos yuxtapuestos que reflexionan. En un primer nivel de sofisticación de estos sistemas, están diseñados y creados para responder, en este juego dinámico, a partir de una codificación computacional escrita estáticamente, donde se predice que las interacciones se agotan, pero se arreglan, aunque el sistema mismo ser dinámico en su funcionamiento. Pero en otro nivel más complejo, los sistemas involucrados en la interacción pueden incluir subsistemas de aprendizaje y evolución, lo que hace que el resultado sea impredecible hasta que los interactuadores y los objetos comiencen a interactuar. El equilibrio entre control y sorpresa es parte de lo que él llama estética interactiva, y es el 
papel del artista diseñar este equilibrio, que es una parte importante de su responsabilidad para diseñar interacciones y sus efectos. En la conclusión del texto, Rokeby afirma que, en lugar de los objetos definitivos ya preparados, quienes trabajan con medios interactivos deben ser conscientes de que crearán más y más relaciones, y la capacidad de representar relaciones y efectos de manera funcional será una fundamental competencia para que los artistas (o diseñadores) se expresen.

Jonas Löwgren y Erik Stolterman, autores cuyo texto Thoughtful Interaction Design (2004) es contemporáneo al de Moggridge (2006), se centra en un análisis de los elementos de pensamiento y razonamiento de este nuevo diseñador para Digital, con muchas referencias a la teoría de Schön. (1983) Al comienzo de su libro, otro autor, Pelle Ehn, al presentar el trabajo, hace una declaración sorprendente de que la diferencia entre este diseñador y los diseñadores que se preocupan por el diseño para Arquitectura o productos industriales es en el hecho de que la actividad dirigida al objeto digital está más cerca de hacer una película o una obra de teatro que de diseñar una taza. Afirma que el diseñador de productos digitales necesita trabajar en lo que él llama Gestalt dinámico, haciendo referencia y dando otro significado al concepto de Gestalt, el principio de los fundamentos más importantes del diseño. El concepto estaría intrínsecamente relacionado con el hecho de que Interaction Design se ocupa de la desmaterialización del diseño, tomando una definición de la materia a partir de sus propiedades físicas, estando sujeto a nuevas lógicas de manipulación, visualización, transformación. La actividad de proyección y el objeto final del diseño se fusionan sin precedentes. No hay separación ni diferencia del material que subyace al diseño y la manipulación de las formas y propiedades estéticas hechas por el software a partir de la realización y existencia de los objetos creados en su forma final, ya que todos están hechos de la misma sustancia, comparten la misma base: códigos. Un elemento de interfaz creado en el software de Diseño dentro del proceso de Diseño ya está potencialmente compuesto de la misma sustancia que el elemento de interfaz funcional final. Aunque los procesos de creación e implementación segregados aún persisten, ambos dentro de las máquinas están hechos del mismo código, el mismo lenguaje. La creación se fusiona con el logro. Esto se nota en la evolución de los pasos de creación de prototipos digitales, que hacen cada vez más difícil distinguir que los prototipos son solo prototipos, ya que las alternativas pueden, en teoría, nacer y manipularse libremente en su estado final de realización. Lo digital disuelve los límites entre lo material y lo virtual en este tipo de desarrollo, y esta es, por lo tanto, la segunda propiedad fundamental que hace que Interaction Design aparezca como una ruptura.

En un trabajo seminal sobre lo que él llama el Lenguaje de los Nuevos Medios, Lev Manovich (2001) se ocupa de describir las características de este nuevo lenguaje, las propiedades de este material que reside dentro de las máquinas computacionales y la forma en que se trata, haciendo una discusión amplia sobre las nuevas formas de creación, que se profundizan en el texto posterior llamado Software Takes Command (Manovich, 2013). El impacto principal en esta compresión del tiempo, donde el diseño y el objeto final comparten la misma materialidad bajo las leyes de la lógica digital y computacional, es que crean las condiciones potenciales para la manipulación, alteración y reacción de los objetos y la acción de los diseñadores sobre los objetos, en tiempo real. Todas las formas y 
soluciones son, en el límite, solo contextuales, el resultado de un momento en el tiempo y la conjunción de variables de entorno temporales, recursos tecnológicos, acción interactiva y acción del diseñador al manipular o definir el comportamiento de estas variables. La discusión sobre la materialidad digital en el Diseño (o desmaterialización - física cuando digital) adquiere otra dimensión cuando pensamos en el desarrollo y la adopción masiva de nuevas tecnologías digitales de experiencia e interacción inmersiva y realidades simuladas como la Realidad Virtual y Realidad Aumentada. Se está haciendo un esfuerzo significativo para desarrollar e invertir en entornos de Realidad Aumentada o Virtual en los que se crea un universo de navegación sensorial, totalmente digital e interactivo, regido por las leyes de digitalización y sin ninguna limitación física del mundo real. No solo se fusionan el proceso de diseño y el objeto final, sino también los entornos de inmersión que simulan el mundo físico. Y aunque contienen referencias visuales, estéticas y formales a los artefactos o elementos de la naturaleza del mundo físico, las interacciones y los objetos con los que interactúan tienen libertad para modificar estos espacios y artefactos.

La ruptura de lo digital, a partir de sus dos elementos primordiales - la interacción y la desmaterialización del Diseño - una vez más ejerce la deconstrucción y reconstrucción de los fundamentos del diseño, probando sus límites. Convergente en los textos de los diversos autores de Interaction Design es la idea de que esta disciplina se basa en los principios, métodos e ideas que se originan en el Diseño, presentes en Arquitectura, Diseño Industrial, Diseño Gráfico, caracterizados, como hemos visto, por una tradición de rigidez, racionalismo, búsqueda de soluciones definitivas e ideas de forma. Esto puede verse como paradójico para las lógicas que gobiernan la interacción y la desmaterialización, no solo como propiedades sino también como potencialidades. Quizás por esta razón, se observa más recientemente en esta trayectoria de Diseño aplicado a lo digital, un agotamiento del marco de disciplina para lidiar con la tecnología requerida y las evoluciones de escala, así como el enfoque y la definición del problema. Por otro lado, también hay en la desmaterialización una devaluación indirecta de la forma, ya que no solo la forma sigue a la función, sino que son los patrones y sistemas de funciones, y su orquestación de acciones y reacciones, los que se convierten en la esencia del Diseño de Interacción. Esto se hará cada vez más evidente a medida que las interfaces de voz conversacionales se vuelvan primordiales entre las personas y las máquinas: los objetos se volverán invisibles, dejando solo su existencia funcional-lógica.

\section{La ruptura del material: materia activa y la lógica de la invasión digital del mundo físico.}

La cuarta y última ruptura descrita aquí - que, incluso en sus etapas iniciales, parece bastante prometedora - proviene de la investigación de lo que llamaremos materia activa, en referencia al término adoptado por uno de los centros de estudio de la asignatura, el Self-Assembly Lab, de MIT, organizador de la reunión de Active Matter Summit. Con su primera edición en 2014, el evento tuvo como objetivo reunir a investigadores que todavía estaban dispersos y que habían publicado o discutido poco sobre los avances en estudios y experimentos con materia activa en los últimos años. Según lo descrito por Leila Kinney 
en la introducción del trabajo del mismo nombre, Active Matter, publicado en 2017, estos investigadores tenían básicamente dos objetivos: la miniaturización de la máquina, como si fueran dispositivos electrónicos para ser utilizados a nivel celular e inspirados por la idea de que las proteínas son como máquinas autónomas en su funcionamiento básico; y la oportunidad de aplicar este tipo de desarrollo al diseño y fabricación de objetos, brindando oportunidades sin precedentes a arquitectos, artistas y diseñadores. Citado en el texto, el investigador Neri Oxman defendió en una conferencia en esta primera edición de la reunión que creó un entorno único de convergencia de áreas y habilidades que se describen a continuación: diseño computacional, fabricación aditiva, ingeniería de materiales científicos y biología sintética (Kinney en Tibbits, 2017). Esta unión de Arte, Arquitectura, Diseño, Ciencia, Informática e investigación se atribuye a las ideas visionarias de György Kepes cuando asumió la presidencia del MIT, a fines de la década de 1960. Húngaro, vinculado a la Bauhaus, emigró también a Chicago (New Bauhaus) en el la década de 1940, llegó al MIT en 1946, y en las décadas siguientes fue el principal articulador y pensador del trabajo interdisciplinario propuesto entre los campos del Arte, la Ciencia y la Ingeniería en los campos de investigación conjunta que hoy caracteriza esencialmente la investigación realizada en el MIT Media Lab. Una audacia en ese momento, el exitoso modelo ha sido seguido por otros centros de investigación líderes en todo el mundo.

En una definición precisa, la materia activa (Active Matter) es un campo de estudio emergente que se centra en el desarrollo de materiales físicos que tienen propiedades tales como: la capacidad de componerse, transformarse, sentir, reaccionar y ser programado, y procesarse computacionalmente a partir de la información que reciben de dentro o fuera (Tibbits in Tibbits, 2017). El concepto puede extenderse a diferentes escalas, desde microscópico (a nivel celular) hasta macroscópico (como construcciones arquitectónicas activas), así como cruzar la intersección de diferentes disciplinas, desde la Biología hasta la Informática. Tibbits hace una distinción importante entre la definición de materia activa y materia programable, que en algunos textos se toman como sinónimos. Para el autor, son diferentes niveles de evolución del concepto y la investigación. La materia programable se define como una etapa más limitada y temprana del campo, cuando se pensaba que estos materiales eran solo elementos físicos incluso de chips, computadoras y baterías conectadas en miniatura. Las materias activas, por otro lado, son puramente materia porque incluyen en su propia composición física las propiedades descritas anteriormente que los hacen programables, transformables, adaptables y combinables. Dado que están pensadas y creadas bajo estas premisas a nivel celular, no se limitan a formas, tamaños, propiedades o aplicaciones restringidas y estandarizadas. Por el contrario, brindan la libertad de diseñar y crear personalizaciones que pueden aplicarse y desarrollarse para cualquier propósito y escala, así como dirigirse en su composición para realizar un conjunto dado de estas acciones o propiedades. El autor afirma que la evolución del campo nos llevará a un punto de inflexión sobre cómo nos relacionamos con los materiales, y compara este momento con lo que hemos estado viviendo en la informática durante algunas décadas, cuando ya no se limita a los mainframes, y ha ganado escala y penetración en nuestra vida diaria, transformando completamente la relación que teníamos con la tecnología y las computadoras. Nuestra relación con los materiales de hoy supone que son transitables, inertes, como si fueran esclavos de las herramientas que utilizamos para componer- 
los y hacerlos lo que queremos. En este nuevo paradigma, utilizaremos propiedades que existían solo en el mundo digital/virtual (o, como llamamos anteriormente, en entornos desmaterializados) que ahora también se aplican al mundo físico, como una premisa de las características de los materiales y la forma en que los tratamos. La materia queda sujeta a la lógica computacional, reprogramación, reconfiguración, corrección de errores, montaje y desmontaje. Complementando, por otro lado, las propiedades del mundo que conocemos como naturales y orgánicas, se pueden aplicar a materiales sintéticos como crecimiento, reparación, mutación, replicación. Por lo tanto, creamos la posibilidad de producir artefactos físicos a partir de materiales dotados de inteligencia que modifican sus características, estructura o comportamiento debido a estímulos internos, externos o una cierta programación.

Como ejemplo de esta potencialidad, los autores incluyeron en una edición limitada del trabajo antes mencionado una primera página producida en materia activa, dotada con un patrón de pequeños triángulos irregulares que reaccionaron dinámicamente cuando un haz de luz alcanzó su superficie reflectante, volviendo a su estado natural tan pronto como la luz se estaba desvaneciendo.

Con contornos que todavía parecen ser ciencia ficción, un mundo con producción avanzada y adopción masiva de artefactos en materia activa es uno donde, desde un simple comando o reacción programada a un contexto ambiental, los objetos se reconfigurarían para adaptarse mejor a su uso, y que pueden obtener diferentes formas y propiedades estéticas para el gusto y la necesidad humana, o incluso para las decisiones de inteligencia y adaptación intrínsecas al material mismo. Tales especulaciones aparecen en artículos sobre el tema de los centros de futurología como Singularity University ${ }^{2}$, o la revista de tecnología en línea de $\mathrm{IEEE}^{3}$, la organización profesional más grande del mundo para ingeniería y ciencias aplicadas. DARPA4, la agencia de investigación avanzada de la agencia de defensa de los Estados Unidos, conocida popularmente como el lugar de nacimiento de Internet, ha estado desarrollando un programa sobre el tema, en asociación con universidades como Harvard, MIT y Cornell, desde mediados de 2008. Otra universidad estadounidense, Carnegie Mellon, invierte en asociación con el gigante tecnológico Intel en la creación de lo que llamaron Claytronics, nanorobots de tamaño molecular con propiedades computacionales y de autocomposición que pueden formar cualquier objeto, también reconfigurando su forma y propiedades estéticas bajo el mando de programadores o interactores.

\section{Conclusión: ¿un nuevo paradigma para el Diseño?}

En la historia, el Diseño se ha construido, deconstruido y reconstruido, a partir de lo que fue concebido por Walter Gropius, a principios del siglo pasado, hasta llegar hoy a la materia activa.

Cada ruptura presentadaaquí constituye una pieza fundamental de este proceso evolutivo, confrontando los ideales y funciones originales lanzados por los preceptos y prácticas de Bauhaus. Las rupturas también se relacionan entre sí, en un proceso conflictivo, a veces confrontando ciertos aspectos, a veces refiriéndolos, implícita o explícitamente. 
Como hemos visto, en la dimensión teórica, los autores han refutado el racionalismo y la búsqueda de la universalización; en la dimensión práctica, los profesionales y los investigadores desafiaron hacerlo y pensar en hacerlo, involucrándose en la tecnología, alejando al diseñador de la manipulación directa del objeto y revelando nuevas formas de mirar; lo digital ha traído lo que antes podía parecer impensable: la interacción como la esencia de los proyectos que se transforman y reaccionan como premisa y que tienen como norma la desmaterialización; y finalmente, la impasibilidad de la materia misma ha dado paso a nuevos conceptos y posibilidades, volviéndola voluble, variable, viva y activa.

Desde una perspectiva futura, estos movimientos nos proporcionan tanto una base teórica como una práctica-procesal que sugiere un proceso de Diseño totalmente dinámico, asumiendo como premisa un objeto final de este Diseño que también es dinámico, ya sea digital o físico. La velocidad de llegada y masificación de los objetos digitales ha transformado la realidad física en un entorno de realidad simulada o mixta. En teoría, la fusión computacional-biológica a nivel atómico-celular de las materias activas, que permite el desarrollo de materiales que reaccionan y se transforman en sus propiedades y formas, parametrizables y sensibles, dará lugar a cualquier objeto que supongamos hoy como estático. En cualquiera de estas formas hay un cambio fundamental en la esencia de lo que se espera de la creación y realización de lo artificial que sirve a la sociedad.

¿Y qué viene después?

En un llamado por una nueva visión del diseño en su papel en el mundo, los autores Anthony Dunne y Fiona Raby han escrito un manifiesto titulado Diseño Especulativo (Dunne, Raby, 2013), en el que llaman la atención sobre la necesidad de vislumbrar el Diseño de un mundo nuevo, si realmente queremos resolver los problemas de este que habitamos. En contraste con lo que critican como una actividad adictiva que trata de pensar lo nuevo a partir de lo establecido, instan a los diseñadores a pensar en el Diseño como la herramienta capaz de crear este nuevo entorno, entendiendo el contexto en el que trabajan en sus creaciones como futuros inexistentes, especulativos.

Sin embargo, desde nuestro punto de vista, el mundo de las materias activas y las realidades virtuales, y la especulación sobre futuros inexistentes, desconocidos e imponderables exigen no solo técnicas y métodos actualizados, o incluso nuevas herramientas, sino un nuevo propósito y un nuevo enfoque. El diseño tiene el desafío de dar paso a un nuevo paradigma que renueva el propósito del Diseño en su amplitud. Diseño que es, por excelencia, la respuesta al impulso de pretender ser, una característica que, como Simon (1996) definió, es parte de la esencia del carácter transformador y creador de la realidad del ser humano, y que constituye su actividad motriz.

Es por eso que nuestra propuesta en este artículo es diferente, aunque complementaria. Además de utilizar el Diseño - como disciplina y como un conjunto de prácticas y teorías - para especular sobre nuevas realidades, nuestra intención es especular sobre qué Diseño se necesitará en una nueva realidad.

Y esta nueva realidad - a juzgar por la velocidad con la que avanzan el procesamiento de la información, la simulación de la realidad y la fusión de las tecnologías de la biología y la informática - más que simplemente imponderable y desconocida, sigue siendo totalmente impredecible. Sobre lo que se convertirá en nuestra realidad en el futuro, aunque en el futuro cercano, solo estamos seguros de que requerirá numerosos nuevos conocimientos, 
características, habilidades y herramientas, y un marco conceptual completamente nuevo. Por lo tanto, pretender ser -estar continuamente adaptándose y transformándose para supropia supervivencia - es, en nuestra opinión, el nuevo paradigma del Diseño.

Un diseño autónomo, sensible, inteligente y resiliente, un diseño que pulsa, respira, se transforma y se adapta, tratando de sobrevivir a las condiciones más adversas y que no termina cuando se le da alguna forma, sino que solo nace allí, para transformarse continuamente, como es la vida.

\section{Notas}

1. Kandisnsky creía que los impresionistas sentaron las bases de esta ciencia del arte, pero lo hicieron inconscientemente. El trabajo a realizar a partir de entonces fue asumir esto conscientemente.

2. Recuperado de: https://singularityhub.com/2018/04/29/stuff-3-0-the-era-of-programmable-matter/. Accedido en junio de 2019.

3. Recuperado de: https://spectrum.iee.org/robotics/robotics-hardware/make-yourown-world-with-programmable-matter. Accedido en junio de 2019.

4. Recuperado de: https://www.afcea.org/content/programmable-matter-research-solidifies. Accedido en junio de 2019.

\section{Listas de Referencias bibliográficas}

Archer, B. (1979). Design as a Discipline. Design Studies, 1(1), pp.17-20.

Buchanan, R. (1992). Wicked Problems in Design Thinking. Design Issues, 8(2), pp. 5-21.

Cardoso, R. (2008). Uma introdução à história do design (3 ed). São Paulo, SP: Blucher.

Cardoso, R. (2016). Design para um mundo complexo. São Paulo, SP: Ubu Editora.

Cooper, A.; Reimann, R.; Cronin, D.; \& Noessel, C.. (2014). About face: the essentials of interaction design (4 ed). Indianapolis, IN: Wiley.

Droste, M. (2013). Bauhaus: 1919-1933. (Casa das Línguas, Trans.). Köln, Germany: Taschen.

Dunne, A.; \& Raby, F. (2013). Speculative everything: design, fiction and social dreaming. Cambridge, MA: The MIT Press.

Forty, A. (2007). Objetos de desejo: design e sociedade desde 1750. (P. M. Soares, Trans.). São Paulo, SP: Cosac Naify.

Flusser, V. (2013). O mundo codificado: por uma filosofia do design e da comunicação. (R. Cardoso, Org.). (R. Abi-Sâmara, Trans.). São Paulo, SP: Cosac Naify.

Garret, J. J. (2002). The elements of user experience: user-centered design for the web. Berkeley, CA: New Riders.

Hebron, P. (2016). Machine learning for designers. Sebastopol, CA: O’Reilly Media.

Heller, S.; \& Pettit, E. (2013). Design em diálogo: 24 entrevistas por Steven Heller e Elinor Pettit. (C. Knipel, Trans.). São Paulo, SP: Cosac Naify.

Jonson, S. (2001). Cultura da interface. Rio de Janeiro, RJ: Jorge Zahar Ed. 
Kandinsky, W (2012). Ponto e linha sobre plano (2 ed). (E. Brandão, Trans.). São Paulo, SP: Editora WMF Martins Fontes.

King, R.; Churchill, E. F.; \& TAN, Caitlin. (2017). Designing with data: improving the user experience with $A / B$ testing. Sebastopol, CA: O'Reilly Media.

Löwgren, J.; Stolterman, E. (2004). Thoughtful interaction design: a design perspective on information technology. Cambridge, MA: The MIT Press.

Lupton, E.; \& Miller, J. A.(2006). The abc's of the Bauhaus and design theory. London, England: Thames \& Hudson.

Lupton, E.; \& Phillips, J. C. (2008). Novos fundamentos do design (2 ed). São Paulo, SP: Cosac Naify.

Manovich, L. (2001). The language of new media. Cambridge, MA: The MIT Press.

Manovich, L. (2013). Software takes command. New York, NY: Bloomsbury Academic.

Meggs, P. B.; \& Purvis, A. W. (2016). History of graphic design (6 ed). Hoboken, NJ: Wiley.

Moggridge, B. (2006). Designing interactions. Cambridge, MA: The MIT Press.

Morville, P.; \& Rosenfeld, L. (2002). Information architecture for the world wide web: designing large-scale web sites. Cambridge, MA: O'Reilly, 2002.

Negroponte, N. (1969). Toward a theory of architecture machines. Journal of Architectural Education, 23(2), pp. 9-12.

Negroponte, N. (2003). From soft architecture machines. In: N. Wardrip-Fruin, \& N. Monfort (Eds), The New Media Reader (pp. 354-366). Cambridge, MA: The MIT Press.

Nesbitt, K. (Org.). (2013). Uma nova agenda para a arquitetura: antologia teórica (19651995). (V. Pereira, Trans.). São Paulo, SP: Cosac Naify.

Norman, D. (2013). Design of everyday things - revised and expanded edition. New York, NY: Basic Books.

Oxman, R. (2017). Thinking difference: theories and models of parametric design thinking. Design Studies, 52(C), pp. 4-39.

Pavliscak, P. (2015). Data-informed product design. Sebastopol, CA: O’Reilly Media.

Poynor, R. (2010). Abaixo as regras: design gráfico e pós-modernismo. (M. Bandarra, Trans.). Porto Alegre, RS: Bookman

Rittel, H. W. J.; \& Webber, M. M. (1973). Dilemmas in a general theory of planning. Policy Sciences, 4(2), pp. 155-169.

Rokeby, D. (1995). Transforming mirrors: subjectivity and control in interactive media. In: S. Penny (Ed). Critical issues in electronic media (pp. 133-158). Albany, NY: State University of New York Press.

Saffer, D. (2010). Designing for interaction: creating innovative applications and devices. Berkeley, CA: New Riders.

Schumacher, P. (2004). Responsive environments - from drawing to scripting. Recuperado de: http://www.patrikschumacher.com/Texts/AADRLDrawingScripting.html

Schumacher, P. (2009). Parametricism: a new global style for architecture and urban design. Recuperado de: https:/www.patrikschumacher.com/Texts/Parametricism\%20-\%20A\%20 New\%20Global\%20Style\%20for\%20Architecture\%20and\%20Urban\%20Design.html

Schumacher, P (2012). Parametric semiology - the design of information rich environments. Recuperado de: http://www.patrikschumacher.com/Texts/Design\%20of\%20Information\%20Rich\%20Environments.html 
Schön, D. (1983). The reflective practitioner: how professionals think in action. New York, NY: Basic Books.

Simon, H. A. (1996). The sciences of the artificial (3 ed). Cambridge, MA: The MIT Press. Shedroff, N. (1994). Information interaction design: a unified field theory of design. Recuperado de: http://nathan.com/information-interaction-design-a-unified-field-theoryof-design

Smith, G. C. (2006). What is interaction design. In: B. Moggridge. Designing interactions (pp. IX-XIX). Cambridge, MA: The MIT Press.

Soddu, C. (2002). New naturality: a generative approach to art and design. In: Leonardo, 35(3), p. 291-294.

Tibbits, S (2017). Active matter. Cambridge, MA: The MIT Press.

Turkle, S. (2005). The second self: computers and the human spirit. 20th anniversary edition. Cambridge, MA: The MIT Press.

Wingler, H. M. (1976). The Bauhaus: Weimar, Dessau, Berlin, Chicago. Cambridge, MA: The MIT Press.

Winograd, T.; \& FLORES, Fernando. (1986). Understanding computers and cognition: a new foundation for design. Norwood, NJ: Ablex Publishing.

\title{
Agradecimientos
}

Queremos expresar nuestro agradecimiento al Programa de Pós-graduação em Design de PUC-Rio, Brasil. Este trabajo se realizó con el apoyo de la Coordenação de Aperfeiçoamento de Pessoal de Nível Superior - Brasil (CAPES) - Código de Financiamiento 001.

\begin{abstract}
If we take as starting point the emergence of the Bauhaus school, Design as a discipline - a conjunction of theory, practice and education - completes, in 2019, a century of existence. Over that time, it has evolved into a fundamental element of contemporary society, marked by mass production and consumption, urbanization, and the revolution in information and communication technologies. Its original essence and function - to plan and shape all the artificial things that humanity creates, guided by a rationalist and deterministic idea, in search of definitive solutions - had to be transformed to meet the demands of society. More recently, the demand for contextualization, digital media, and the development of so-called active matter point to even deeper disruptions. In this paper, we make a historical and critical review of Design, and - from four turning points - we propose a new look at the most fundamental aspects of the discipline and its challenges for the future.
\end{abstract}

Keywords: Design - active matter - digital - society - transformation - ideology. 
Resumo: Se considerarmos o surgimento da escola Bauhaus como ponto de partida, o design como disciplina - uma conjunção de teoria, prática e educação - completa, em 2019, um século de existência. Durante esse período, tornou-se um elemento fundamental da sociedade contemporânea, marcada pela produção e consumo em massa, pela urbanização e pela revolução nas tecnologias da informação e comunicação. Sua essência e função originais - planejar e moldar todas as coisas artificiais que a humanidade cria, guiadas por uma idéia racionalista e determinista, em busca de soluções definitivas - tiveram que ser transformadas para satisfazer as demandas da sociedade. Mais recentemente, a demanda por contextualização, mídia digital e o desenvolvimento da chamada matéria ativa apontam para rupturas ainda mais profundas. Neste artigo, fazemos uma revisão histórica e crítica do design e, a partir de quatro pontos de inflexão, propomos uma nova visão dos aspectos mais fundamentais da disciplina e de seus desafios para o futuro.

Palavras chave: Design - matéria ativa - digital - sociedade - transformação - ideologia.

[Las traducciones de los abstracts fueron supervisadas por el autor de cada artículo] 
